\title{
Über die Entwicklung der Dottersackzirkulation bei Scyllium stellare.
}

\author{
Von \\ F. Hochstetter in Innsbruck.
}

Hierzu Tafel XXXVII.

Die Beobachtungen, über welche ich im nachfolgenden berichten will, wurden in den Jahren 1902 und 1903 gemacht, als ich dank dem liebenswürdigen Entgegenkommen des Vorstandes der zoologischen Station in Triest, des Herrn Prof. Cori, den Tersuch unternehmen konnte, lebend nach Innsbruckübersandte ScylliumEier im Seewasseraquarium zur Weiterentwicklung zu bringen, um mir auf diese Weise, da ich wegen Zeitmangels einen längeren Aufenthalt am Meere nicht nehmen konnte, selbst eine grössere Zahl von Scylliumembryonen der verschiedensten Entwicklungsstadien möglichst sorgfaltig konservieren zu können.

In der Tat gelang der Versuch in jeder Beziehung. Der grösste Teil der übersandten Eier kam lebend in Innsbruck an und ich vermochte einzelne von ihnen durch zwei Monate, bis Mitte Juli lebend zu erhalten, bis die altesten Embryonen eine Lange ron 30-32 mm hatten. Professor Cori sandte mir einzelne Eier, die sich noch im Furchungsstadium befanden, ferner eine grössere Zahl mit ganz jungen Embryonalanlagen und endlich aber auch solche, deren Embryonen bereits äussere Kiemen besassen. In allen Fällen erfolgte die Weiterentwicklung ohne Störung. Erst im Juli, als die Temperatur auch in unserem Keller, in dem ich das Seewasseraquarium aufgestellt hatte, eine etwas höhere wurde, starben einzelne Embryonen ab, wodurch ich veranlasst wurde, die letzten noch lebenden Embryonen rasch aufzuarbeiten. Gewiss wăre es mit Hilfe einer Kühlvorrichtung und durch Erneuerung des Seewassers möglich gewesen, eine Anzahl von Eiern noch länger am Leben zu erhalten. Da ich jedoch über die Entwicklungsstadien, deren ich für meine Untersuchungen bedurfte, bereits verfügte, so hatte ich keine Veranlassung, Versuchen in dieser Richtung weitere Zeit zu opfern.

Beim Konservieren der Embryonen konnte ich nun ohne besondere Schwierigkeit die einzelnen Phasen der Entwicklung der 
Dottersackzirkulation am lebenden Objekte beobachten und entweder selbst abbilden, oder von einem Zeichner abbilden lassen.

Wenn ich nun über die dabei gemachten Beobachtungen berichte, so bin ich mir wohlbewusst, dass ich nicht viel neues $z \mathfrak{u}$ bringen vermag, nachdem $\mathrm{H}$. Virchow in zwei kurzen, in der Gesellschaft naturforschender Freunde in Berlin gehaltenen Vorträgen fast alles gesagt hat, was über den Gegenstand zu sagen war. Virch ow hat aber keine seine Angaben illustrierenden Abbildungen veröffentlicht und dadurch wird ein Teil seiner Angaben eigentlich nur für denjenigen vollkommen verständlich, der mindestens ein oder das andere Entwicklungsstadium der Dottersackzirkulation von Scyllium zu sehen Gelegenheit hatte. Ich selbst wenigstens konnte mir nach seinen Angaben, als ich den Abschnitt über die Dottersackzirkulation der Selachier für Hertwigs Handbuch schreiben sollte, kein klares Bild über die Verhaltnisse bei Scyllium machen und war deshalb gezwungen mich hauptsächlich auf die die Dottersackzirkulation von Pristiurus betreffenden Angaben und Abbildungen von Balfou $r$ (1) zu beziehen. Erst als ich einige Stadien der Dottersackzirkulation von Scyllium aus eigener Anschauung kennen gelernt hatte, war es mir möglich, Virchows Ausführungen $\mathrm{zu}$ verstehen.

Ich halte es deshalb nicht für überflüssig, wenn ich auf der dieser Mitteilung beigegebenen Tafel eine grössere Zahl von möglichst naturgetreuen Abbildungen veröffentliche, welche dem Beschauer die einzelnen Phasen der Entwicklung der Dottersackzirkulation von Scyllium stellare von dem Momente des ersten Auftretens von mit Blut gefüllten Gefässen in dem ausserembryonalen Teile des Keimes, bis zu dem Zeitpunkte vor Augen führen, in welchem der ganze Dottersack von Gefässen bedeckt ist. Der Text meiner Mitteilung soll lediglich dazu bestimmt sein, eine etwas ausführliche Erklärung der Bilder zu bieten.

Figur 1 zeigt uns den Embryonalpol eines Scylliumeies, dessen Blastoderm einen Durchmesser von nicht ganz $10 \mathrm{~mm}$ besass, dessen Embryonalanlage mit ihrem Schwanzende das Blastoderm um etwa $0,7 \mathrm{~mm}$ überragte und 34 Ursegmente erkennen liess. Der Gefăssbezirk des Blastoderms erscheint durch die Embryonalanlage in zwei schmale Felder von sichelförmiger Gestalt geteilt, deren konvexer Rand dem kaudalen Rande des Blastoderms entspricht, während ihr konkaver Rand jederseits von 
einem Blutgefässe eingenommen wird, in welchem wir die Anlage der Dottersackarterie erkennen. In dem Felde selbst sind deutlich eine grössere Zahl von Blutinseln wahrzunehmen.

Dieses früheste Entwicklungsstadium, in welchem am lebenden Selachierei deutlich ein Gefăssbezirk auf dem Dottersacke wahrzunehmen ist, und welches Balfo ur (1) noch nicht gekannt hatte, hat Virchow für Embryonen von Pristiurus richtig beschrieben und als Stadium 1 bezeichnet. Von einer Dottersackzirkulation kann jedoch in diesem Entwicklungsstadium schon aus dem Grunde noch nicht gesprochen werden, weil die beiden Anlagen der A. omphalomesentericae. weder untereinander, dort wo sie aus dem Embryo hervorzukommen scheinen, noch auch mit der Aorta des Embryos in Verbindung stehen. Es sind also diese Anlagen an Ort und Stelle selbst und nicht etwa durch Auswachsen eines der Embryonalanlage angehörigen Gefässes (Aorta) entstanden.

Virchow spricht sich bezüglich dieses Punktes in seiner ersten Mitteilung (7) dahin aus, dass seine Befunde dafür sprechen, dass die Anlage der Dottersackarterien im Dottersackbindegewebe selbst und nicht durch Auswachsen von der Embryonalanlage aus entsteht. In seiner zweiten Mitteilung dagegen (8) lässt er die Frage, ob die Arterien durch Auswachsen vom Embryo aus, oder in loco entstehen, noch offen, wenn er sagt: „Die primitive Arterie wird bei Scyllium zunächst in der Năhe des Embryos sichtbar, wie auch eine Figur von Balfour angibt. Dies scheint für ein Auswachsen derselben vom Herzen zu sprechen. Ich will nicht behaupten, dass dieser Schluss zwingend sei, wenn wir aber zu diesem Schlusse gedrängt werden, dann müssten wir annehmen, dass Abschnitte des primären Gefässbezirkes auf dem Dottersacke der Selachier durch Auswachsen vom Herzen entstehen, während andererseits die Blutinseln im ganzen Umfange des Mesodermfeldes in loco entstehen. Hiermit scheint mir ein noch nicht gelöstes Problem der Untersuchung gekennzeichnet."

In dem kaudalen konvexen Rande des Gefässbezirkes ist weder jetzt noch auch in den nächstfolgenden Entwicklungsstadien bei der Betrachtung des frischen Objektes irgend etwas von einem grösseren Gefăsse zu sehen, welches der paarigen Anlage der Dottersackvene entsprechen würde. Doch konnte ich an der Querschnittserie durch eine Lumbryonalanlage mit 34 Ursegmenten, bei welcher die beiden Subintestinalvenen bereits entwickelt waren 
und sich unmittelbar kaudal von der kaudalen Darmpforte zu dem bekannten von P. Mayer (4) und Rabl (5) beschriebenen, ganz kurzen Venensack vereinigen, nachweisen, dass in diesen Sack von beiden Seiten her je ein grösseres aus dem Rande des Gefässbezirkes kommendes:Gefăss einmündet. Zweifellos handelt es sich in diesen beiden Gefässen um die Anlagen der paarigen Dottersackvenen. Dabei war ich aber bei diesem Embryo nicht in der Lage, festzustellen, ob eine von den beiden (die linke), oder gar beide Venen, in welche der Herzschlauch kaudalwarts übergeht und die Rabl (5) als V. omphalomesentericae bezeichnet mit dem Subintestinalvenensacke in Verbindung stehen. Möglicherweise ist ein solcher Zusammenhang in diesem Entwicklungsstadium doch schon vorhanden und war wegen mangelhafter Ausdehnung des betreffenden Gefässabschnittes nur an meiner Serie nicht nachzuweisen, denn auch bei älteren Embryonen, bei denen die V. omphalomesenterica sinistra sicher schon mit dem Subintestinalvenensacke in Verbindung steht, habe ich ihn hăufig nicht nachweisen können. Ob er aber von vorne herein da ist, sobald die erste Anlage der paarigen Subintestinalvene kaudal von der hinteren Darmpforte in Form hohler Gefässröhren nachzuweisen ist, möchte ich nach den Befunden an Schnittserien durch Embryonen mit 30, 31 und 32 Ursegmenten entschieden bezweifeln.

In Fig. 2 ist die Ansicht der Embryonalpolseite eines Eies wiedergegeben, dessen Embryo eine Länge von $5 \mathrm{~mm}$ hatte. Das Blastoderm hat den Dotter bis über den Äquator des Eies hinaus umwachsen und die beiden Hälften des kaudalen Blastodermrandes, die hier bereits eine deutliche, wenn auch noch schwache braunrote Färbung zeigen, vereinigen sich ventral vom Schwanzende des Embryos unter spitzem Winkel und sind wenigstens auf eine kurze Strecke weit schon miteinander verwachsen. Es hat also in diesem Entwicklungsstadium die Bildung der sogenannten Dottersacknaht bereits begonnen.

Die den Gefässbezirk gegen den gefässfreien Teil des Blastoderms abgrenzenden beiden Dottersackarterien sind nun schon sehr viel deutlicher sichtbar als in dem Stadium der Figur 1. Dabei hat sich ihr Verlauf in zweierlei Weise geändert. Erstlich unabhăngig von der Verschiebung des Blastodermrandes, indem sie zwar noch an derselben Stelle aus der Embryonalanlage hervorzukommen scheinen, wie früher, aber nunmehr bereits einen seine Konvexität 
kranialwärts und nach aussen kebrenden Bogen bilden. Die zweite Veränderung, die ihren peripheren Verlaufsabschnitt betrifft, ist durch die Verschiebung des Blastodermrandes bedingt, indem die Arterien, nachdem sie den eben geschilderten Bogen gebildet haben, kaudalwärts dem Blastodermrande zustreben, um ihm entlang, sich ihm gleichzeitig immer mehr nähernd, zu verlaufen. Jede von den beiden Arterien zeigt also einen S-förmigen Verlauf.

In diesem Entwicklungsstadium steht die rechte Dottersackarterie bereits durch zwei im Gebiete der Vorniere gelegene Gefässröhren (es sind die ersten auftretenden Vornierenarterien Rückerts und Rabls) mit der Aorta in Verbindung. Dagegen lassen sich solche Verbindungen zwischen linker Arterie und Aorta nicht nachweisen und auch mit der rechten Dottersackarterie ist die linke noch nicht in Verbindung getreten. Sie endet also, gegen die Embryonalanlage verfolgt, an der linken Wand der vorderen Darmpforte blind.

Der jüngste Embryo bei dem ich die Verbindung zwischen der rechten Dottersackarterie und der Aorta nachweisen konnte, besass 36 Ursegmente. Von den beiden dem Blastodermrande folgenden, der Embryonalanlage zustrebenden Venen, die sich in den Subintestinalvenensack ergiessen, ist auch jetzt bei der Betrachtung des lebenden Blastoderms noch nichts wahrzunehmen. Das gleiche gilt übrigens auch noch für das in Fig. 3 und 4, sowie für das in Fig. 5 abgebildete Entwicklungsstadium.

Fig. 4 zeigt uns wieder ein Scylliumei in der Ansicht von oben, dessen Embryo eine Länge von $6,1 \mathrm{~mm}$ hatte und dasselbe $\mathrm{Ei}$ ist in Fig. 4 bei etwas schwächerer Vergrösserung von der kaudalen Seite her abgebildet worden. Die letztere Figur lässt erkennen, dass das Blastoderm den Dotter schon zum grössten Teile umwachsen hat, dass sich die Dottersacknaht dem in Fig. 2 abgebildeten Stadium gegenüber beträchtlich verlängert bat und dass der Blastodermrand ein annăhernd elliptisches Feld umgrenzt, im Bereiche dessen der Dotter noch unbedeckt zutage tritt. Dabei zeigt sowohl die Dottersacknaht, als auch der Blastodermrand jene eigentümliche braunrote, zum Teil ins orange übergehende Färbung, auf welche Virchow (8) als erster aufmerksam gemacht hat. Der von den Dottersackarterien begrenzte Gefässbezirk des Blastoderms hat sich im Bereiche der Embryonalanlage sehr erheblich verbreitert und umgreift mit seinen beiden 
dem Blastodermrande folgenden kaudalen Ausladungen den unbedeckten Teil des Dotters zangenförmig. Dabei hat sich die Konvexităt des Bogens, welchen jede von den beiden Dottersackarterien bildet, etwas vorgeschoben, ohne jedoch das Niveau des Kopfendes der Embryonalanlage ganz zu erreichen.

Ausser diesen beiden Dottersackarterien sind, obwohl sie sicher schon bestehen, bei der Betrachtung des lebenden Objektes noch keine anderen Gefässe auf eine Iängere Strecke hin durch den Gefässbezirk zu verfolgen. Ob bei dem zu dem Ei der Fig. 3 und 4 gehörigen Embryo die beiden Dottersackarterien in ihrer ventral vom Herzen gelegenen Verlaufsstrecke bereits $\mathrm{zu}$ einem in der Medianebene gelegenen unpaaren Stamme verschmolzen waren, habe ich, da der betreffende Abschnitt des Blastoderms bei der Einbettung in Paraffin lädiert worden war, nicht mit Sicherheit feststellen können. Sicher aber ist, dass dies bei etwa gleichalten Embryonen (mit 52 Ursegmenten) bereits der Fall ist, bei denen sich ferner auch noch feststellen liess, dass sich die linke Dottersackarterie auch noch über die Verschmelzungsstelle mit der rechten hinaus in kaudaler Richtung bis an die linke Wand der vorderen Darmpforte verfolgen liess, wo sie dann blind endigte.

Bei Pristiurus scheint nun, wie dies aus der Beschreibung, welche Virchow (7) auf pag. 99 von seinem Stadium 2 gibt, hervorgeht, die Umwachsung des Dotters durch das Blastoderm wesentlich langsamer vor sich zu gehen wie bei Scyllium, indem bei der ersten Form die Dottersackarterie schon einen kurzen unpaaren Stamm bildet, während bei Scyllium, wenn das Blastoderm den Dottersack so weit umwachsen hat, wie in dem Stadium 2 Virchows, von einem solchen Stamme noch nichts wahrzunehmen ist (vergl. meine Fig. 2). Damit stimmt auch überein, dass der zu dem Stadium 2 Virchows gehörige Pristiurusembryo bereits 57 Ursegmente besass, während ich bei einem Embryo von Scyllium dessen Blastoderm ähnliche Verhältnisse darbot, wie die des in Fig. 3 und 4 abgebildeten Eies, 52 Ursegmente zählen konnte. Aus diesem Umstande erklärt sich auch die von Virchow bei der Beschreibung seines ein Blastoderm von Scyllium betreffenden Stadiums 3 aufgestellte Behauptung, dass der Embryo in diesem Stadium weiter vorne sitze als im Stadium 2, und dass infolge davon der unpaare Stamm 
der Dottersackarterie bedeutend kürzer sei, als in diesem Stadium, eine Behauptung, welche in dieser Fassung gewiss nicht richtig ist. Vielmehr ist der unpaare Stamm der Dottersackarterie bei Scyllium in dem Stadium 3 der Umwachsung des Dotters durch das Blastoderm noch bedeutend kürzer als bei Pristiurus im Stadium 2 der Umwachsung, weshalb im Stadium 3 der Umwachsung an Scylliumeiern der Embryo weiter vorne zu sitzen scheint, als im Stadium 2 der Umwachsung an Pristiuruseiern. Jedenfalls konnte ich an den von mir untersuchten Scylliumeiern eine mit der Umwachsung des Dotters durch das Blastoderm vollkommen parallel gehende gleichmässige Verlängerung des Dottersackarterienstammes beobachten (vergl. Fig. 5-8).

Die vom Blastoderm unbedeckte Stelle des Dotters verschwindet nun bald vollständig, indem sich die Blastodermrănder entsprechend der Dottersacknaht aneinanderlegen. Diese Aneinanderlagerung ist bereits bei Embryonen mit 55 Ursegmenten vollzogen. Die Fig. $5 \mathrm{a}$ und $\mathrm{b}$ zeigen die Ansichten der beiden Hălften eines zu einem solchen Embryo gehörigen Dottersackes, der durch eine den Embryo in seiner Mitte quer durchschneidende Ebene in zwei gleiche Halften geteilt gedacht ist, wobei Fig. 5 a die zu dem Kopfteile, Fig. 5b die zu dem Schwanzteile des Embryos gehörige Hälfte des Dottersackes dastellt, wăhrend der Embryo selbst in der Zeichnung nicht mit aufgenommen wurde.

Die Dottersackarterie bildet nun schon einen, auch bei der einfachen Betrachtung des Blastoderms erkennbaren, kurzen, ventral von dem vorderen Körperabschnitte des Embryos in der Medianebene verlaufenden einfachen Stamm (Fig. 5a), der, wie ich schon früher hervorgehoben habe und wie auch Virchow richtig erkannt hat, durch Verschmelzung der früher noch paarigen Anfangsabschnitte der beiden Dottersackarterien entstanden ist. Dieser Stamm gabelt sich ventral von dem Kopfende des Embryos in die beiden den Gefasssbezirk des Blastoderms umgrenzenden Arterien. Der Gefässbezirk selbst erscheint im Bereiche der Embryonalanlage breit schildförmig, läuft aber (Fig. 5b) auf der dem Schwanzende des Embryo angehörigen Hälfte des Dottersackes in eine Zunge aus, in deren Mitte, die durch ihre Färbung ausgezeichnete Dottersacknaht gelegen ist. $\mathrm{Zu}$ beiden Seiten dieser Naht aber verlaufen, wie dies die Schnittserie lehrt, denn 
am frischen Blastoderme sind sie immer noch nicht sichtbar, die beiden Dottersackvenen, die sich an der Stelle, an welcher sie die Embryonalanlage erreichen, zu einem kurzen in die Subintestinalvene einmündenden Stamm vereinigen. Jetzt sind auch am frischen Blastoderme bereits kleine aus den Dottersackarterien hervorgehende Gefåssreiserchen wahrnehmbar.

Erst an dem lebend untersuchten Ei eines Embryos mit $2 \mathrm{~mm}$ Stirn-Scheitelhöckerlänge (vergl. Fig. 6a und b) konnte ich jedoch diese Gefässreiserchen mit voller Deutlichkeit durch den ganzen Gefässbezirk hindurch verfolgen und sehen, wie sie in die jetzt auch schon deutlich hervortretenden, zu beiden Seiten der Dottersacknaht verlaufenden Dottersackvenen einmündeten. Diese beiden Venen waren übrigens bereits auf eine längere Strecke weit, in dem Bereiche, in welchem anschliessend an die Embryonalanlage die Dottersacknaht schon verschwunden ist (vergl. Fig. 6b), zu einem unpaaren Stamme vereinigt.

Der Gefässbezirk selbst hat sich gegenüber dem Stadium der Fig. 5a und b nicht unerheblich vergrössert, indem er nun mit seinem breiten Teile den Dottersack beinahe bis zu seinem Äquator bedeckt, während sein die Dottersacknaht umschliessender zungenförmiger Abschnitt, der ebenfalls etwas breiter geworden ist, sich gegen den dem Embryonalpole gegenüberliegenden Pol des Dottersackes vorgeschoben hat. Dabei haben sich die beiden, zwischen dem breiten und dem zungenförmigen Abschnitte des Gefässbezirkes gelegenen, einspringenden Winkel verkleinert. Ich will hier noch besonders hervorheben, dass $\mathrm{Vir}$ chow die in meinen Fig. 5 and 6 wiedergegebene Form des Gefässbezirkes schon vollkommen richtig beschrieben hat.

Wie nun der Dottersack schliesslich vollständig vom Gefässbezirke umwachsen wird, das zeigen die Figuren 7, 8 und 10 anf das klarste. Diese Figuren zeigen den Dottersack in denselben Ansichten wie die Figuren 5 und 6 und lassen erkennen, wie sich der unpaare Dottersackarterienstamm in dem Maße immer weiter verlängert, in welchem sich der Gefässbezirk an der Kopfhălfte des Dottersackes über seinen Äquator allmählich herabschiebt, während sich gleichzeitig (Fig. 7b) die Gefässbezirkszunge, nachdem die Dottersacknaht vollständig verschwunden ist, mit ihrer Spitze dem unteren Pole des Dottersackes nähert. Dabei ist durch die Verschmelzung der beiden früher zu beiden Seiten der Dotter- 
sacknaht vorhanden gewesenen Dottersackvenen ein einheitlicher Venenstamm entstanden.

Indem sich nun das Ende der Gefässbezirkszunge über den unteren Pol des Dottersackes gegen dessen Kopfhälfte zu vorschiebt und sich so dem vorderen Rande des auch an der Vorderseite des Dottersackes noch weiter herabgewachsenen Gefässbezirkes nähert, legen sich die Seitenränder dieser Zunge an die Seitenränder des an diese Zunge anschliessenden breiten Teiles des Gefässbezirkes an und es verschmelzen die diese sich aneinanderlegenden Randabschnitte einfassenden Abschnitte der Dottersackarterien miteinander. Dadurch werden zwei grössere Arterienäste gebildet, welche nunmehr als direkte Fortsetzungen der beiden ursprünglichen Dottersackarterien erscheinen. Diese Arterien (vergl. Fig. 8b) steigen über die kaudale Hälfte des Dottersackes schief gegen die unpaare Dottersackvene auf. Zweifellos sind aber diese beiden Arterien, wie sie uns in Figur $8 \mathrm{~b}$ entgegentreten, durchaus nicht ihrer ganzen Länge nach durch Verschmelzung der den Rand des Gefässbezirkes im Gebiete zwischen Zunge und breitem Teile konturierenden Arterienabschnitte entstanden. Vielmehr entsteht durch diesen Verschmelzungsprozess nur der zentrale Teil des Arterienstammes jeder Seite und dieser verlangert sich erst sekundar in der Richtung gegen die unpaare Dottersackvene durch Ausweitung gewisser Gefässbahnen der Dottersackwand. In ähnlicher Weise und nicht bloss durch einfaches Längenwachstum verlängert sich wohl auch die Dottersackvene.

In dem Stadium der Fig. 8 ist bereits der ganze Dottersack bis auf einen seiner Kopfhälfte angehörigen, schmalen, transversalen, seitlich spitzwinkelig begrenzten Streifen (Fig. 8a) von einem Gefässnetze bedeckt. Aber auch dieser Streifen verschwindet bald gänzlich und es zeigen die Dottersackgefassse nunmehr Verhältnisse, wie sie in den Figuren 10a und $b$ wiedergegeben sind. Wăhrend aber, soweit ich wenigstens sehen konnte, in den Stadien der Figuren 6 und 7 die beiden Äste, in welche sich der Dottersackarterienstamm gabelt, den vorderen Rand des Gefässbezirkes einfassten, sah ich, wie dies in Fig. $8 \mathbf{a}$ wiedergegeben ist, zu der Zeit, in welcher nur noch ein ganz schmaler Streifen des Blastoderms der Gefässe entbehrt, anschliessend an die Teilungsstelle der Dottersackarterie und an ihre beiden Äste eine ganz schmale, von zarten Gefässen durchzogene, und von einem ebensolchen 
Gefässe begrenzte Zone den vorderen Rand des Gefässbezirkes bilden und dieser Rand ist es, der sich dann mit dem Rande der vorgeschobenen Gefässbezirkszunge vereinigt.

Nicht immer aber entwickelt sich der Gefässbezirk auf dem Dottersacke in so symmetrischer Weise. Vielmehr kommen, wie dies auch schon Virchow (8) angegeben hat, Assymmetrien geringeren oder höheren Grades recht häufig zur Beobachtung. Vor allem werden gröbere Störungen der Symmetrie in denjenigen Fällen zu beobachten sein, in denen, wie dies Virchow (8) zuerst angegeben hat, und wie ich dies anch für einige von mir untersuchte Eier bestătigen konnte, die Dottersacknaht nicht einen einfachen median sagittal eingestellten Streifen darstellt, sondern sich gabelt. Ich vermag aber natürlich nicht $z u$ entscheiden, ob alle gröberen Assymmetrien in der Ausbildung des Gefassbezirkes, die ich zu beobachten Gelegenheit hatte, auf eine solche Gabelung der Dottersacknaht zurückgeführt werden können. Dass aber wenn, solche Assymetrien zur Ausbildung kommen, die Umwachsung des Dottersackes durch den Gefässbezirk nicht in so regelmässiger Weise erfolgen kann, wie dies gewöhnlich der Fall zu sein scheint, ist jedenfalls sicher.

In Fig. 9 habe ich die Kopfhălfte eines Scylliumdottersackes abgebildet, bei welchem die Umwachsung durch den Gefässbezirk in hochgradig unregelmässiger Weise erfolgt war. Und zwar betraf die Unregelmässigkeit nur die linke Halfte des Dottersackes, sodass, wenn die Ursache dieser Unregelmåssigkeit, was sich ja nicht mehr feststellen liess, was ich aber gerade in diesem Falle für wahrscheinlich halte, in einer Gabelung der Dottersacknaht bestanden hatte, angenommen werden muss, dass der eine Ast der Gabel genau median sagittal eingestellt gewesen war, während der andere Ast nach der linken Seite hin abgewichen sein mochte.

Aus den im vorhergehenden gemachten Angaben erhellt, dass sich die Dottersackzirkulation bei Scyllium stellare im wesentlichen in ganz ähnlicher Weise ausbildet wie bei Pristiurus, auf welche Form sich die allgemein bekannten Angaben Balfours beziehen. Was die von diesem Autor veröffentlichten Abbildungen anbelangt, so scheinen mir dieselben allerdings mehr den Wert von Schemen, als den von naturgetreuen Bildern $\mathrm{zu}$ besitzen. Dies dürfte vor allem für seine Fig. 5 (Pl. 8) gelten. Aber auch bezüglich seiner Figuren 2 und 3 spricht manches dafür, dass 
sie nicht genau den natürlichen Verhältnissen entsprechen, so vor allem der Umstand, dass in Fig. 2 der unpaare Stamm der Dottersackarterie langer erscheint als in der Fig. 3, welche ein älteres Stadium darstellt, was bei den Eiern, welche dem Zeichner zur Vorlage gedient hatten, wohl kaum der Fall gewesen sein dürfte. Recht gut stimmen dagegen die in Balfours Fig. 4 zum Ausdrucke gebrachten Verhăltnisse mit denen überein, welche meine Figuren 8a und b wiedergeben. Balfour hat diese Figur, in der sowohl die Gefässe der Kopfhälfte, als die der Schwanzhälfte des Dottersackes abgebildet sind, als eine "Diagrammatic projection " bezeichnet und treten an derselben vor allem die beiden an meiner Fig. 8a sichtbaren Dottersackarterienäste, welche über die Schwanzhälfte des Dottersackes gegen die Dottersackvene konvergierend aufsteigen, deutlich hervor und ebenso jene dünne Arterie, welche diese beiden Äste entsprechend dem kaudalen Rande der Gefässbezirkszunge miteinander verbindet. Und gerade diese Figur scheint mir zu beweisen, dass sich der Gefässbezirk von Pristiurus, auch was seine Form anbelangt, in ganz ăhnlicher Weise entwickeln und über den Dottersack ausdehnen dürfte, wie der von Scyllium und dass die Figuren 2 und 3 von Balfour als Schemen zu betrachten sein werden. Leider hatte ich selbst keine Gelegenheit, Pristiuruseier 'auf die Verhältnisse der Entwicklung der Dottersackzirkulation zu untersuchen, hoffe aber, dass die im vorhergehenden gemachten Bemerkungen andere Forscher, denen Pristiurusmaterial zur Verfügung steht, dazu veranlassen werden, sich darüber zu ăussern, ob die auf Grund meiner Beobachtungen an Scylliumeiern über Balf o urs Figuren ausgesprochenen Vermutungen zutreffend sind oder nicht. Sehr interessant wäre es auch zu erfahren in welcher Weise sich bei Selachierformen, bei denen wie bei Torpedo die Umwachsung des Dotters durch das Blastoderm sehr viel langsamer erfolgt, wie bei Pristiurus und Scyllium, der Gefässbezirk über den Dottersack ausbreitet und schliesslich denselben vollständig umschliesst.

Innsbruck, am 3. März 1905. 


\section{Literaturverzeichnis.}

1. B a 1 f o u r, F. M.: A Monograph on the Development of elasmobranch Fishes. London 1878.

2. H of $\mathrm{mann}$, C. K.: Entwicklungsgeschichte des Herzens nnd der Blutgefässe bei den Selachiern. Morpholog. Jahrbuch Bd., 19. 1893.

3. Leydig, F.: Beiträge zur mikroskopischen Anatomie und Entwicklungsgeschichte der Rochen nnd Haie. Leipzig 1852.

4. Ma y e r, P.: Über die Entwicklung des Herzens und der grossen Gefässstämme bei den Selachiern. Mitt. a. d. zool. Station zu Neapel, Bd. 7. $1886-1887$.

5. Rabl, C.: Über die Entwicklung des Venensystemes der Selachier. Festschrift zum 70. Geburtstage L e u k a rts. Leipzig 1892.

6. R ü c k e r t, J.: Über die Entstehung der endothelialen Anlage des Herzens und der ersten Gefässstämme bei den Selachierembryonen. Biologisches Centralblatt, Bd. 8. 1888.

7. Virchow, H.: Über die Entwicklung des Gefäsșbezirkes auf dem Selachierdottersacke. Sitz.-Ber. d. Ges. natarf. Fr., Berlin 1895, Nr. 5.

8. Derselbe: Über Dottersacknaht und primären Kreislauf bei Scyllium. Ebenda, 1897, Nr. 5.

9. Derselbe: Über Blutinseln und Gefässbezirk von Torpedo ocellata. Ebenda, 1889, Nr. 5.

10. Z i egle r H. E. und F.: Beiträge zur Entwicklungsgeschichte von Torpedo. Arch. f. mikrosk. Anatomie, Bd. 39, 1892.

\section{Erklärung der Figuren auf Tafel XXXVII.}

Die Figuren 1-3 stellen Ansichten des Dottersackes von Scyllium stellare bei der Betrachtung von oben her dar. Vergr. 3,5 fach. Die betreffenden Embryonen besassen eine Länge von 3,8 mm (Fig. 1), $5 \mathrm{~mm}$ (Fig. 2), und $6,1 \mathrm{~mm}$ (Fig. 3). Fig. 4 zeigt bei 2,5 facher Vergrösserung den Dottersack der Fig. 3 in der Ansicht von hinten (kaudale Hälfte des Dottersackes.

Die folgenden Figuren zeigen Ansichten der Dottersäcke älterer Embryonen, und zwar ist in den mit a bezeichneten Figuren, sowie in der Figur 9, die Kopfhälfte, in den mit $b$ bezeichneten Figuren die Schwanzhälfte des Dottersackes bei 2,5 facher Vergrösserung wiedergegeben.

Der zu dem Dottersacke der Fig. 5a und $b$ gehörige Embryo besass 5ว́ Ursegmente und hatte eine Stirn-Scheitelhöckerlänge von $1,9 \mathrm{~mm}$, die zu den Dottersäcken der folgenden Figuren gehörigen eine Stirn-Scheitelhöckeriänge von $2 \mathrm{~mm}$ (Fig. 6a und b), von 2,1 mm (Fig. 7a und b), respektive eine Gesamtlänge von $24,6 \mathrm{~mm}$ (Fig. $8 \mathrm{a}$ und b), von $24,5 \mathrm{~mm}$ (Fig. 9), und von $28 \mathrm{~mm}$ (Fig. 10a und b). 